\title{
The application of Scilab software in frequency mode simulation on the circular membrane
}

\author{
Dwi Nova Siti Handayani ${ }^{1, *)}$, Yudhiakto Pramudya ${ }^{1, *)}$, Suparwoto ${ }^{2}$, Muchlas ${ }^{1}$ \\ ${ }^{1}$ Program Studi Magister Pendidikan Fisika Universitas Ahmad Dahlan, Jalan Pramuka No 42, \\ Sidikan, Umbulharjo, Yogyakarta 55161 \\ 2 Program Pascasarjana Universitas Negeri Yogyakarta, Jalan Colombo No 1, Karangmalang, \\ Caturtunggal, Depok, Sleman, DIY 55281 \\ ${ }^{*}$ Email: yudhiakto.pramudya@pfis.uad.ac.id and dwinovasiti.05@gmail.com
}

Received 24 August 2018, Revised 5 September 2018, Published 30 September 2018

\begin{abstract}
Scilab software is utilized for solving physics cases in two dimensional wave equations. This study aims to obtain a frequency mode simulation on the circular membrane so that it can display the wave pattern of each frequency mode. The method is to compare the simulation results with the normal mode circular membrane in each mode in to the theoretical calculation. The simulation has been done by utilizing the physics concept in a wave equation involving $\cos \theta$. The function of the wave equation is $\zeta(r, \theta, t)=A J(k r) \cos m \theta \cos \omega t$, assuming that $\mathrm{r}$ is the radius of the circle membrane. The value of the vibration frequency mode in theoretical are $\mathrm{f}_{01}$ $=(200.6 \pm 2.0) \mathrm{Hz}, \mathrm{f}_{11}=(319.7 \pm 3.1) \mathrm{Hz}, \mathrm{f}_{21}=(428.5 \pm 4.2) \mathrm{Hz}$. By using the Scilab program application, the results obtained show that the results frequency mode simulation $\mathrm{f}_{01}, \mathrm{f}_{11}$, dan $\mathrm{f}_{21}$ are similar to the normal mode in theoretical calculation. Thus, the simulation can be used to visualize waves of two dimensions in vibration and wave learning.
\end{abstract}

Keyword: Scilab, circular membrane, simulation, vibration

\section{Introduction}

In the science, there are interesting phenomena that have not been discussed in vibrating objects in the case of two-dimensional equations. For example, the phenomena of vibrating objects in musical instruments with circular membranes. The vibrations of drums, ketipung, and tambourines are the interesting phenomenon to be investigated. Vibration frequency on a circular membrane depends on the tension and type of membrane. If the membrane vibrates at a particular frequency, the modes of vibration will appear. The modes of vibration that appear depend on the position of the point on the membrane.

Number of scientific literature has discussed the completion of wave equations on the rectangular membranes and the circular membranes which generally present a standard solution but have not reviewed some other aspects that can occur. Completion of wave equations can use the wave-polynomial method for vibrations that spreads on the rectangular membranes and the circular membranes. 
Vibration mode on the circular membrane with elastic membrane in polar coordinate follows the two dimensional differential equations that can be solved by using the Bessel function. The solution to the wave equation in polar coordinates on a circular object is solved by a variable separation method by the initial conditions and boundary conditions (Javidinejad, 2013). The results show that moving waves derived in exponential form have similar with the cylindrical wave functions (Sahebnasagh et al, 2011).

One effort to understand and reduce the level of difficulty in the study of the twodimensional wave equations concept is by performing an experiment. However, it needs a long time preparation. Hence, it is considered less practical. An alternative model in understanding the concepts and applications of simple and inexpensive two-dimensional wave equations is to do a simulation model. Simulations can be done using a free and open-source software. One of the example is Scilab software.

Scilab software is a software that has a high level programming language. Scilab is similar to MATLAB, Mathematica, and MAPLE which are designed for mathematicalbased computing. MATLAB, Mathematica, and MAPLE are paid software. Scilab is able to display data in the form of $2 \mathrm{D}$ or $3 \mathrm{D}$ graphics so that data can be visualized or displayed into the expected graph or image data. In addition, it also provides relatively complete of the mathematical functions to be used in calculations on the science and engineering. Scilab can be used as a tool to perform differential and integral calculus calculation, linear algebra, statistics, GUI (Graphical User Interface), and others (Campbell et.al, 2000; Sasongko, 2010; Setiawardhana \& Saraswati, 2018: Paulus et al, 2018).

Scilab is an alternative numerical method in computational programming for engineering students (Pires \& Roger, 2002). In science that focuses on basic mathematical concepts, Scilab can be used to test numerical algorithms or calculations (Wenjiang et al, 2009). Scilab also provides many numerical methods. Hence, it is very easy to understand when doing computational programming.

Scilab has been utilized as a simulation tool on the topic of optical geometry (Randjawali, 2017). It simulated the formation of shadows an object. The object has a height of $7 \mathrm{~cm}$ the placed at a distance of $15 \mathrm{~cm}$ in front of a convex mirror that has a radius of $-30 \mathrm{~cm}$. The convex mirror simulated in research is assumed to be a flat surface. From the results of this simulation, obtained the shadow height of $3.5 \mathrm{~cm}$ and the shadow distance of $-7.5 \mathrm{~cm}$. The results of this simulation are compared with the results of analytical calculations obtain an error of $0 \%$, so that the results are the same as the results obtained by analytic methods.

Hence, Scilab is useful software to perfom simulation on physical phenomena. Therefore, the simulation of frequency mode on the circular membrane to visualization of wave can be done by using Scilab Software. 


\section{Theory}

\subsection{The Equation Elastic a Circular Membrane}

System in two-dimensional is a rectangular membrane with dimensions $L_{\mathrm{x}}$ and $L_{\mathrm{y}}$, with fixed the rim, and with tension that is constant throughout surface. To visualize the small element of a membrane that has a two-dimensional surface as shown in Figure 1, several assumptions need to be taken. The membrane is assumed to be thin and perfectly elastic so that displacement is small.

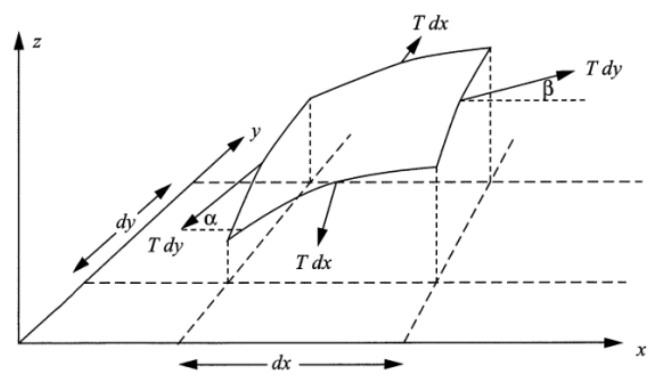

Figure 1. Forces on a rectangular membrane element (Rossing, 2004).

Using Newton's second law, the forces acting on the rim $d x$ have the magnitude $\tau d x$, and their vertical components are $\tau \sin \alpha d x$ and $\tau \sin \beta d x$. Since small $\alpha$ and $\beta$ (Rossing, 2004). Therefore the force on the $y$ component is

$$
F_{y}=\tau d x\left[\left(\frac{\partial \zeta}{\partial y}\right)_{y+d y}-\left(\frac{\partial \zeta}{\partial y}\right)_{y}\right]=\tau d x \frac{\partial^{2} \zeta}{\partial y^{2}} d y
$$

Similarly, the vertical component of the force acting on the rim $d y$ is

$$
F_{x}=\tau d y \frac{\partial^{2} \zeta}{\partial y^{2}} d x
$$

The total force on element $d x d y$ is $F=F_{x}+F_{y}$, so the equation of motion becomes

$$
\tau d x d y\left(\frac{\partial^{2} \zeta}{\partial x^{2}}+\frac{\partial^{2} \zeta}{\partial y^{2}}\right)=\sigma d x d y \frac{\partial^{2} \zeta}{\partial t^{2}} \quad\left(\frac{\partial^{2} \zeta}{\partial x^{2}}+\frac{\partial^{2} \zeta}{\partial y^{2}}\right)=\frac{1}{c_{m}^{2}} \frac{\partial^{2} \zeta}{\partial t^{2}}
$$

this is a wave equation for transverse waves with a speed $c_{\mathrm{m}}$, so that can be written as

$$
c_{m}=\sqrt{\frac{T}{\rho}}
$$

with $c_{m}$ is the sound wave speed on the medium $(\mathrm{m} / \mathrm{s}), \tau$ is the surface tension on the membrane $\left(\mathrm{N} / \mathrm{m}^{2}\right)$, and $\sigma$ is the membrane density $\left(\mathrm{kg} / \mathrm{m}^{2}\right)$.

\subsection{Vibration of a Circular Membrane}

For a circular membrane, the form of the Laplacian in two-dimensional polar coordinates $(r, \theta)$ is (Kinsler, 2000)

$$
\nabla^{2}=\left(\frac{\partial^{2}}{\partial r^{2}}+\frac{1}{r} \frac{\partial}{\partial r}+\frac{1}{r^{2}} \frac{\partial^{2}}{\partial \theta^{2}}\right)
$$


The wave equation on circular membrane becomes

$$
\frac{\partial^{2} \zeta}{\partial r^{2}}+\frac{1}{r} \frac{\partial \zeta}{\partial r}+\frac{1}{r^{2}} \frac{\partial^{2} \zeta}{\partial \theta^{2}}=\frac{1}{c_{m}^{2}} \frac{\partial^{2} \zeta}{\partial t^{2}}
$$

where, $c_{m}{ }^{2}$ is the membrane surface tension divided by the membrane surface density, $r$ is the radius of the surface membrane and $\theta$ is the angle around the axis of the membrane.

Equation (6) can be solved by applying variable separations. Equation (6) becomes

$$
\zeta(r, \theta, t)=R(r) \Theta(\theta) T(t)
$$

substituting equation (7) into equation (6) the wave equation can be written as

$$
\frac{c_{m}^{2}}{R}\left(\frac{d^{2} R}{d r^{2}}+\frac{1}{r} \frac{d R}{d r}\right)+\frac{c_{m}^{2}}{r^{2}} \frac{1}{\Theta} \frac{d^{2} \Theta}{d t^{2}}=\frac{1}{T} \frac{d^{2} T}{d t^{2}}
$$

where, $\omega^{2}=-\frac{1}{T} \frac{d^{2} T}{d t^{2}}$. Because the left and right segments are two functions of different variables. The right-hand side of equation (8) is a two-dimensional differential equation with the periodic function. The similar way can be applied to the second term of the left-hand side (8). Separation variable can be written as (Morse, 1948):

$$
\begin{gathered}
\frac{d^{2} \Theta}{d \theta^{2}}+m^{2} \Theta=0, \Theta(\theta)=\cos (m \theta) \text { or } \Theta(\theta)=\sin (m \theta) \\
\frac{d^{2} R}{d r^{2}}+\frac{1}{r} \frac{d R}{d r}+\left[\left(\frac{2 \pi f}{c_{m}}\right)^{2}-\frac{m^{2}}{r^{2}}\right] R=0
\end{gathered}
$$

with $m$ is integers of $0,1,2, \ldots$

Solving equation (9) which has harmonic solution as follow (Kinsler, 2000)

$$
\Theta(\theta)=\cos (m \theta+\eta)
$$

The $\eta$ is the initial phase angle and takes values of $(2 \pi, 4 \pi, \ldots)$, according to the geometry of the instrument music.

Equation (9) and the wave equation can be written as (Morse, 1948):

$$
\frac{d^{2} R}{d r^{2}}+\frac{1}{r} \frac{d R}{d r}+\left[k^{2}-\frac{m^{2}}{r^{2}}\right] R=0
$$

This is known as Bessel's equation, this relation has the general solution as follow (Kinsler, 2000)

$$
R(r)=A J_{m}(k r)+B \gamma_{m}(k r)
$$

While (12) is the general solution of (13), a membrane that extends across the origin must have the finite displacement at $r=0$. This requires $B=0$ so that

$$
R(r)=A J_{m}(k r)
$$

Application of the boundary condition $R(a)=0$ requires $J_{m}(k a)=0$. A plot of $J_{m}(\mathrm{ka})$ for $m=0,1,2$ is given in Figure 2. And the $n^{\text {th }}$ roots of Bessel function as shown in Table 1.

Table 1. The $n^{\text {th }}$ roots of $J_{\mathrm{m}}(\mathrm{ka})=0$ (Elmore \& Heald, 1969) 


\begin{tabular}{c|cccc}
\hline$j_{\mathrm{mn}}$ & 0 & 1 & 2 & 3 \\
\hline 1 & 2,405 & 3,832 & 5,136 & 6,380 \\
2 & 5,520 & 7,016 & 8,417 & 9,761 \\
3 & 8,654 & 10,173 & 11,620 & 13,015 \\
4 & 11,792 & 13,324 & 14,796 & 16,223 \\
\hline
\end{tabular}

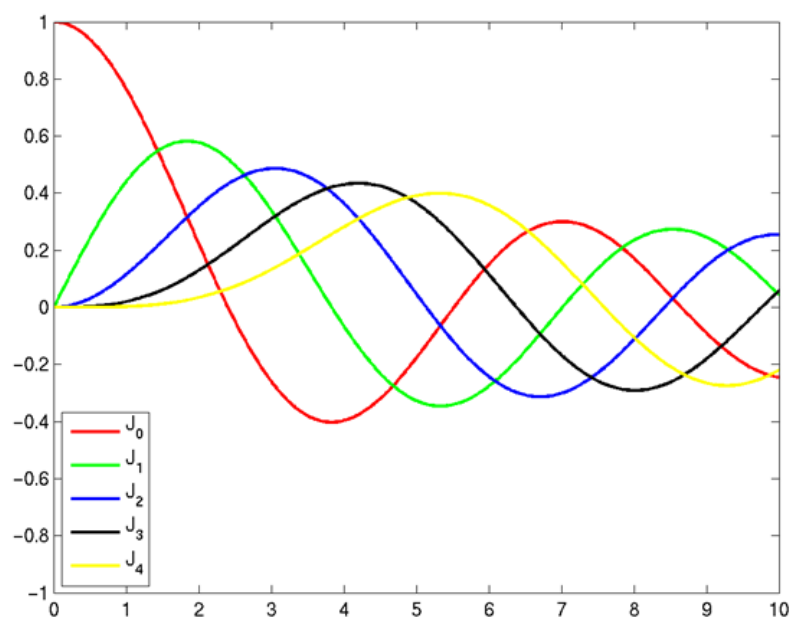

Figure 2. Bessel function of the first kind of order zero, one, and two (Elmore \& Heald, 1969)

If the values of the argument of $J_{m}$ to be equal zero are denoted by $j_{\mathrm{mn}}$, then $k$ assumes the discrete values $k_{m n}=\frac{j_{m n}}{a}$. The membrane radius and the $n^{\text {th }}$ root of the Bessel function are $a$ and $j_{\mathrm{mn}}$, respectively. The general solution in the form equation (7) is

$$
\zeta(r, \theta, t)=J_{m}\left(k_{m n} r\right) \cos (m \theta) \cos (\omega t)
$$

and a relation for the frequency of the system is defined as

$$
f_{m n}=\frac{c k}{\lambda}=\frac{j_{m n} c}{2 \pi a}
$$

where, $f_{\mathrm{mn}}$ is the frequency in vibrating mode, $c_{\mathrm{m}}$ is equal to the speed of the sound wave in medium so that $c_{m}=\sqrt{\frac{T}{\rho}}$, and $a$ is the radius of the membrane.

Some of the possible modes formed of vibration of the circular membrane for small values of $m$ and $n$ as shown in Figure 3 (Elmore \& Heald, 1969). 


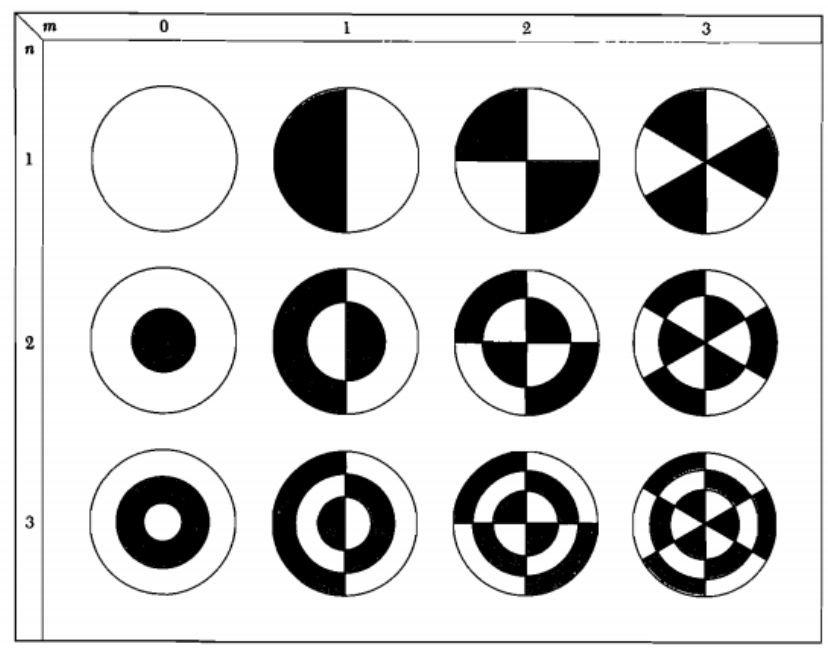

Figure 3. Normal modes of the circular membrane (Elmore \& Heald, 1969)

Figure 3 illustrates some simpler modes of vibration for a circular membrane fixed at the rim. The integer $m$ is determines the number of radial nodal lines and the integer $n$ is determines the number of nodal circle. For example, mode of vibration $(0,1)$ on Figure 3 , in the middle of between the colour black and white is radial nodal line the circular membrane at the rim, it is representing of $m$. The circular membrane forms one nodal circle at the rim, it is representing of $n$.

\subsection{Scilab Software}

Scilab software is software that has a high level programming language. It also provides for the calculations needed in the fields of science and engineering. Scilab in science can be used as a differential and integral calculus calculation, linear algebra, statistics, GUI (Graphical User Interface), and others (Campbell et.al, 2000; Sasongko, 2010; Setiawardhana and Saraswati, 2018: Paulus et al, 2018).

\section{Method}

\subsection{Tools and Materials}

The tools and materials are Scilab 5.52 software which is operated on Lenovo Ideapad S210 netbook with specific Intel Celeron 1037U 1.8 Ghz Processor 2 GB DDR3 320 GB Sata HDD Intel VGA HD Graphic LED 12 Inc Wide Screen.

\subsection{The Prosedure of Research}

\subsubsection{The Frequency Calculation}

The sound wave speed on the medium $\left(c_{m}\right)$ is $(53,41 \pm 0,21) \mathrm{m} / \mathrm{s}$. The radius of the circular membrane $(a)$ is $(0,1019 \pm 0,001) \mathrm{m}$. In this research, the frequency modes used are $f_{01}, f_{11}$, and $f_{21}$ so that the equation (16) can be used to calculate the vibration frequency value. 


\subsubsection{The Vibration Simulation}

The visualization of vibration obtained by using wave equation (15) on the scilab. Then the equation is changed in the Cartesian coordinates. The input parameter are radius of the circular membrane, the value of $m$ and $n$, the value coordinate of $x$ and $y$. All those parameters are inserted to the wave equation two dimensional, and Bessel function. Scilab can display simulation of vibration that it is similar the patterns of vibration on a circular membrane.

\subsection{Algorithm}

Flowchart the research can be seen Figure 4.

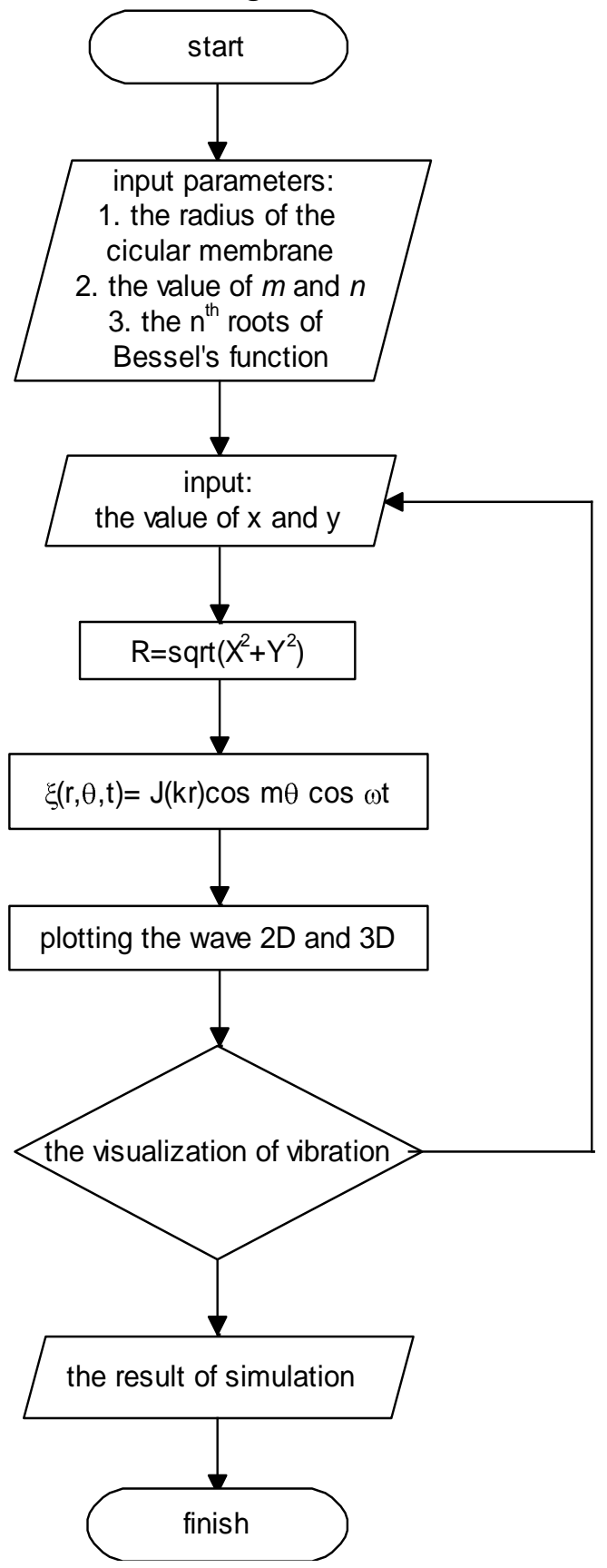

Figure 4. Flowchart of research on the simulation of wave 


\section{Results and Discussion}

The results of the calculation of the vibration frequency by using equation (16) are $f_{01}$ $=(200,6 \pm 2,0) \mathrm{Hz}, f_{11}=(319,7 \pm 3,1) \mathrm{Hz}$, and $f_{21}=(428,5 \pm 4,2) \mathrm{Hz}$. It is the vibration frequency mode value. Normal modes of circular membrane vibrations depend on the values of $m$ and $n$ from the completion of the wave equation.

The solution to the wave equation involves $\cos \theta$ for the function $\Theta$. The boundary conditions which is $\zeta(r, \theta, t)=0$ on the $r=a$ using equation (15) assuming that the value of $t$ is in particular circumstances. For $m=0$ and $n=1$, the value of $\Theta$ is independent of $\theta$. The solution of the wave equation for $\Theta(\theta)$ can be $\sin \theta$ and $\cos \theta$. The value of $x$ and $y$ are changed into Cartesian coordinates so that the visualization of vibration can be seen in Figure 5.

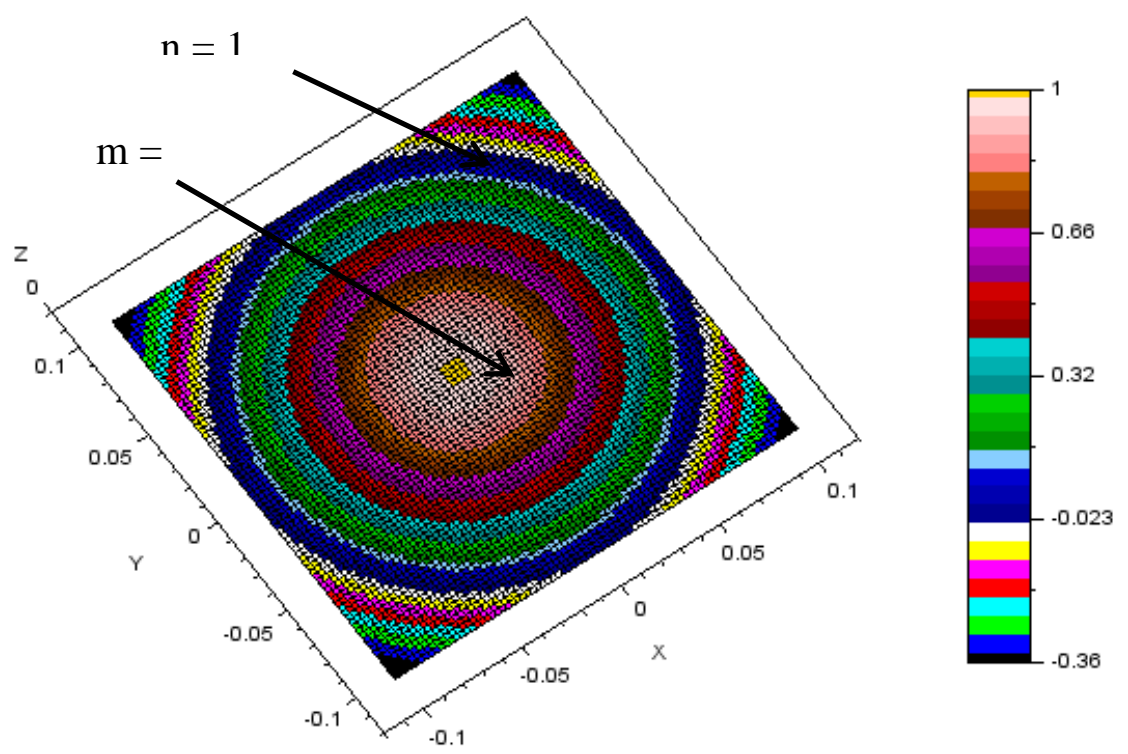

Figure 5. The visualization of vibration for $m=0$ and $n=1$

Figure 5 can be described as the visualization of vibration for $f_{01}$ has a circular wave with a yellow color in the middle representing wave peak and a circular on the outside in dark blue representing circular nodes.

For $m=1$ and $n=1$, the wave equation involves $\theta$ so that $\cos m \theta=0$ with an angle $\theta=\frac{\pi}{2}, \frac{3 \pi}{2}, \ldots$, the solving of the wave equation for $\Theta(\theta) \operatorname{can}$ be $\sin \theta$ and $\cos \theta$, the $n^{\text {th }}$ root of Bessel function, the value of $x$ and $y$ are changed into Cartesian coordinates. The visualization of the mode can be seen in Figure 6 . 


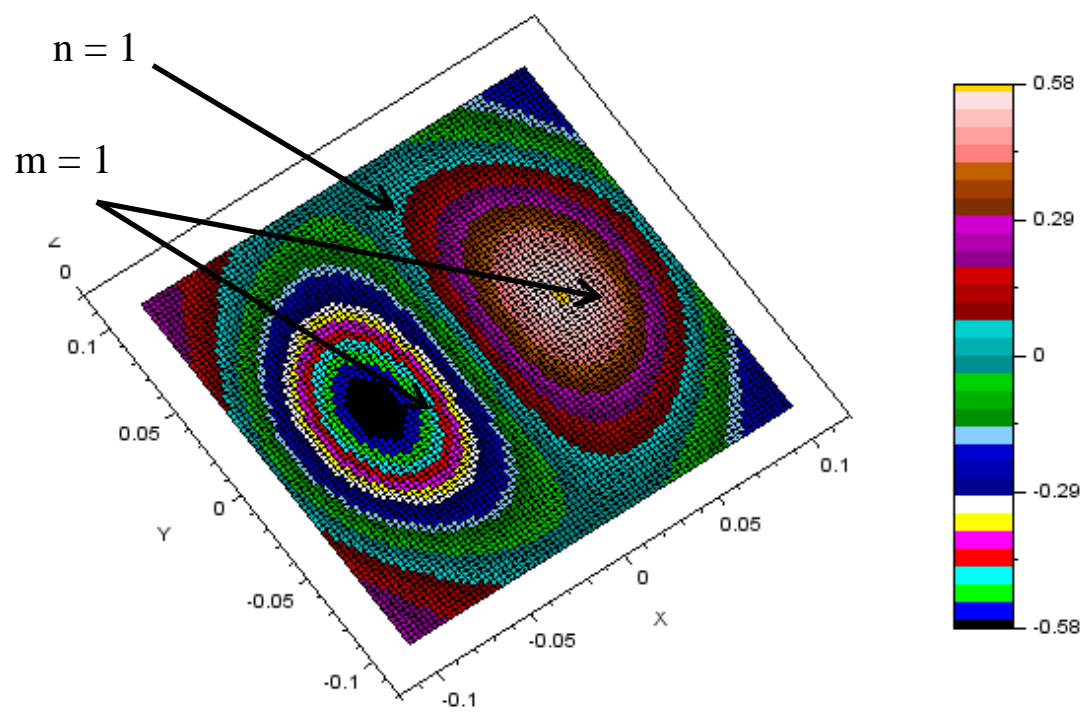

Figure 6. The visualization of vibration for $m=1$ and $n=1$

Figure 6 can be described that the visualization of vibration for $f_{11}$ has two waves that form circular with yellow in the middle representing wave peak and black in the middle representing valley of wave, and circular wave on the outside with light blue representing circular nodes.

For $m=2$ and $n=1$, the wave equation involves $\theta$ so that $\cos m \theta=0$ with an angle $\theta=\frac{\pi}{4}, \frac{3 \pi}{4}, \frac{5 \pi}{4}, \ldots$, the solving of the wave equation for $\Theta(\theta)$ can be $\sin \theta$ and $\cos \theta$, the $n^{\text {th }}$ root of Bessel function, the value of $x$ and $y$ are changed into Cartesian coordinates. The visualization of the mode can be seen in Figure 7.

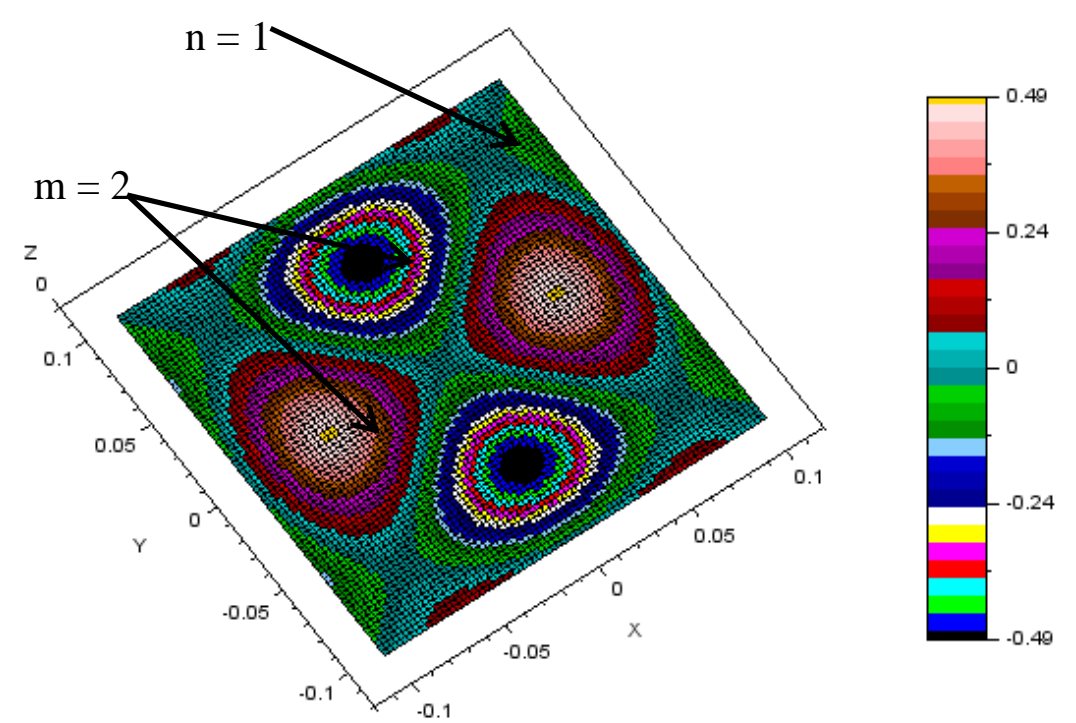

Figure 7. The visualization of vibration for $m=2$ and $n=1$

Figure 7 can be described that the visualization of vibration for $f_{21}$ consists of two waves which are separated in circular with yellow in the middle representing wave 
peak, two waves forming circular with black representing valley of wave, and light blue and green representing circular nodes.

The results of the visualization of vibration in theoretical and the visualization of wave in simulation method using Scilab can be seen in Table 2.

Table 2. The comparison of the visualization of vibration in the theoretical and the simulation

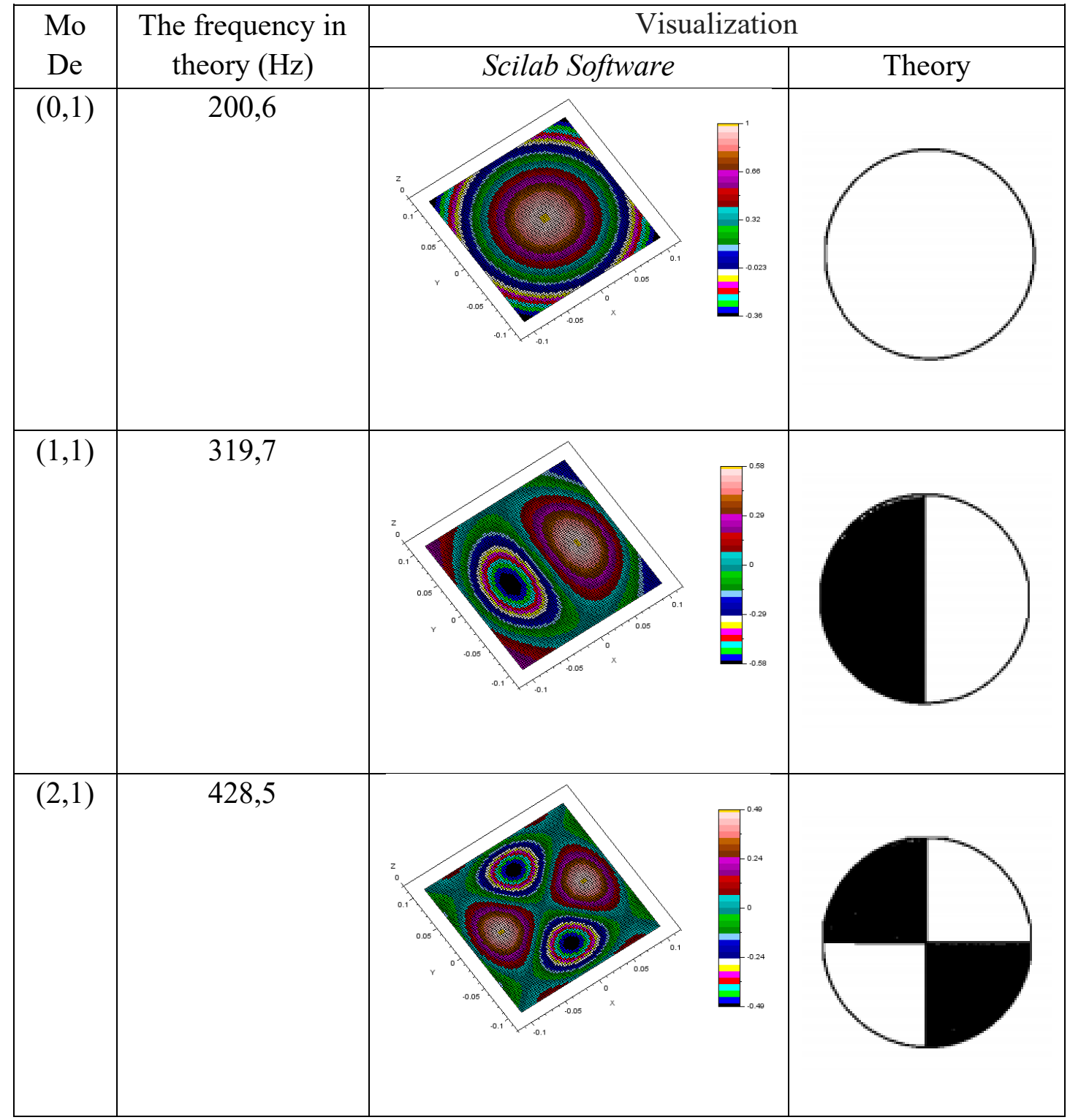

Based on Table 2 in the visualizing waves in theoretical and simulations for frequency modes $f_{01}, f_{11}$, and $f_{21}$ are obtained the visualization results is similar. It can be shown by comparing the surface of circle in theory and simulation using Scilab. Experimentally, the vibration frequency are $f_{01}=(205,0 \pm 0,1) \mathrm{Hz}, f_{11}=(390,0 \pm 0,1)$ $\mathrm{Hz}$, and $f_{21}=(610,0 \pm 0,1) \mathrm{Hz}$ (Handayani, et al, 2018). Despite there is a little discrepancy between the frequency of vibration in experimentally and the theoretical, the visualization is similar. Because the visualization of vibration is obtained from the simulation is similar to the theoretical, then the simulation using Scilab can be used to 
visualize the wave two dimensions on the vibration and wave learning in wave and oscillation course in university level.

\section{Conclusion}

Based on the results of research and discussion on the application of Scilab Software in the frequency mode simulation on the circular membrane, it was concluded that the visualization of wave on theoretical and simulation models with Scilab Software for the frequency modes $f_{01}, f_{11}$, and $f_{21}$ are similar to the theoretical.

\section{Reference}

Campbell, S. L., Chancelier J. P., \& Nikoukhah, R. (2000). Modeling and Simulation in Scilab/Scicos. Springer

Elmore, W. C. \& Heald, M. A. (1969). Physics of Waves. McGraw-Hill: United States of America

Handayani, D. N. S., Pramudya, Y., \& Suparwoto. (2018). Monograf Simulasi Visualisasi Gelombang 2D dan 3D pada Membran Lingkaran dengan Software Scilab. UAD Press: Yogyakarta

Javidinejad, A. (2013). Vibration Modal Solutions Developing Of The Elastic Circular Membrane In Polar Coordinates Based On The Fourier-Bessel Series, Journal of Theoretical and Applied Mechanics, Vol. 43, No. 1, pp. 19-26

Kinsler, L. E., Frey, A. R., Coppens, A. B., \& Sanders, J. V. (2000). Fundamentals of Acoustics, $4^{\text {th }}$ ed. New York: John Willey \& Sons.

Morse, P. M. 1948. Vibration and Sound, $2^{\text {nd }}$ ed. New York: McGraw-Hill

Paulus, E., Suryani, M., Suryana, I., \& Chaerani, D. (2018). Perangkat Komputasi Numerik Scilab Berbasis Open-Source: Algoritma dan Penerapannya. Deepublish Publisher: Yogyakarta

Pires, P. S. M. \& Rogers, D. A. (2002). Free/Open Source Software: An Alternative For Engineering Students. $2^{\text {nd }} A S E E / I E E E$ Frontiers in Education Conference ISBN 0-7803-7444-4/02

Randjawali, E. (2017). Desain Simulasi Pembentukan Bayangan pada Cermin Cembung menggunakan GUI Builder Scilab 5.5.0, Jurnal Penelitian Fisika dan Aplikasinya (JPFA), Vol 7, No 02, Desember 2017 p-ISSN: 2087-9946 eISSN: 2477-1775. http://dx.doi.org/10.26740/jpfa.v7n2.p102-114

Rossing, T. D. \& Fletcher, N. H. (2004). Principles of Vibration and Sound $2^{\text {nd }}$ Edition. Springer: New York

Sahebnasagh, M., Mirjavadi S., \& Bahrami, M. N. (2011). A Novel Wave Approach Solution For Free Vibration Of Circular Membrane Using Conversion Of Standing Wave Into A Moving Wave, Journal of System Design and Dynamics, Vol.5, No. 7, pp. 1508-1517. https://doi.org/10.1299/jsdd.5.1508

Sasongko, S. B. (2010). Metode Numerik dengan Scilab. Andi Offset: Yogyakarta

Setiawardhana \& Saraswati, D. A. (2018). Aplikasi Scilab dengan Arduino. Tira Smart: Tangerang 
Wenjiang, L., Nanping, D., \& Tongshun, F. (2009). Application of Scilab in teaching of Engineering Numerical Computations. IEEE ISBN 978-1-4244-4453-3/09 